\title{
ON METRIC INDEPENDENCE AND LINEAR INDEPENDENCE
}

\author{
L. M. BLUMENTHAL ${ }^{1}$ AND V. L. KLEE ${ }^{2}$
}

For a metric space $M, C M$ will denote the Banach space of all bounded real-valued continuous functions on $M$, with the usual $\|f\|=\sup _{x \in M}|f(x)|$. It is well-known that $M$ is homeomorphic (and, in fact, isometric) with a subset of $C M[4$, p. 543]. We show here that $M$ must be homeomorphic with a linearly independent subset of $C M$. Whether $M$ must be isometric with such a set remains undecided.

Let the distance between two points $x$ and $y$ of $M$ be denoted by $x y$, and for each $x \in M$ let $f_{x}$ be the function $x y \mid y \in M$. The subset $A$ of $M$ is said to be metrically dependent in $M$ provided the family of functions $\left\{f_{a}: a \in A\right\}$ is linearly dependent over $M$. Otherwise, $A$ is metrically independent in $M$. This notion leads quickly to the desired result, by means of the following observations.

(1) If $A$ is a subset of a (not necessarily separable) Hilbert space E, then $A$ is metrically independent in $A$.

Proof. It suffices to show that if $p_{1}, \cdots, p_{n}$ are $n$ distinct points of $A$ and $d$ is the value of the determinant $\left|p_{i} p_{j}\right|_{1, n}$, then $d \neq 0$. But since the subspace of $E$ determined by $\left\{p_{1}, \cdots, p_{n}\right\}$ is a Euclidean space of dimension $\leqq n$, this follows at once by an argument of Schoenberg [5, p. 792]. (See also [2, \$40 and §54].)

(2) If $M$ is a metric space, then $M$ has a bounded homeomorph in which every subset $A$ is metrically independent in $A$.

Proof. By a theorem of A. H. Stone [6], $M$ is paracompact, and hence by a theorem of C. H. Dowker [3, p. 639] there is a Hilbert space whose unit sphere contains a homeomorph of $M$. The desired conclusion then follows from (1).

(3) If $M$ is a metric space, then $M$ is homeomorphic with a linearly independent subset of $C M$.

Proof. In view of (2), we may assume that $M$ is bounded and that every subset $A$ of $M$ is metrically independent in $A$. Now let $T D$ $=f_{x} \mid x \in M$, where $f_{x}$ is as defined above. From the fact that $M$ is bounded and from the definition of metric independence it follows

Presented to the Society, February 26, 1955 ; received by the editors December 9 , 1954.

${ }^{1}$ Fulbright Lecturer, University of Leiden, The Netherlands.

${ }^{2}$ Work on this paper sponsored by the Office of Ordnance Research, U. S. Army, under Contract DA-04-200-ORD-292. 
that $T M$ is a linearly independent subset of $C M$. Since $T$ is an isometry, the proof is complete.

Now if (in the proof of (3)) $T M$ is separable, then so is the linear subspace of $C M$ spanned by $T M$. From a well-known embedding theorem of Banach and Mazur [1, p. 185] there follows

(4) Every separable metric space is homeomorphic with a linearly independent subset of $C[0,1]$.

We do not know ${ }^{3}$ whether every separable metric space is isometric with a linearly independent subset of $C[0,1]$, although it is easy to see that every finite metric space has this property.

Now for an arbitrary metric space $M$, let $K$ be the unit cell $\{f:\|f\| \leqq 1\}$ of the space $(C M)^{*}$ dual to $C M$. Then $K$ is compact in the weak topology, and there is a natural linear isometry of $C M$ into $C K$. Thus we have

(5) For each metric space $M$ there is a compact Hausdorff space $K$ such that $M$ is homeomorphic with a linearly independent subset of $C K$.

We conclude with some remarks on metric dependence.

(6) If a metric space $M$ has fewer than four points, then $M$ is metrically independent in $M$.

A metric quadruple is pseudo-linear $[2$, p. 110] provided each of its triples is linear (i.e., isometric with a subset of $E^{1}$ ), but the quadruple itself is not linear.

(7) For a metric quadruple $Q$, the following three assertions are equivalent:

(i) $Q$ is pseudo-linear.

(ii) The points of $Q$ can be so labelled that $q_{1} q_{2}=q_{3} q_{4}=a>0, q_{2} q_{3}$ $=q_{1} q_{4}=b>0$, and $q_{1} q_{3}=q_{2} q_{4}=a+b$.

(iii) $Q$ is metrically dependent in $Q$.

Proof. That (i) is equivalent to (ii) is noted in $[2$, p. 114]. From (ii) it follows easily that the determinant $\left|q_{i} q_{j}\right|_{1,4}$ is zero, whence (ii) implies (iii). That (iii) implies (i) can be proved by applying results of [2] (in particular, pp. 131 and 293) to the metric transform of $Q$ by the function $\phi(x)=x^{1 / 2}$, but we give here a more elementary proof.

Suppose $Q$ is metrically dependent in $Q$; i.e., $\sum_{1}^{4} a_{i} f_{q_{i}} \equiv 0$ for numbers $a_{i}$ not all zero. Since, by (1), $Q$ is not linear, to show that $Q$ is pseudo-linear it suffices to show that each triple in $Q$ is linear. It is clear that no $a_{i}$ can be different in sign from all the other three, for $f_{q_{i}}$ vanishes only at $q_{i}$. Thus with notation appropriately chosen we

3 Added in proof: An affirmative answer to this question follows from a recent theorem of Arens, to the effect that every metric space is isometric with a closed linearly independent subset of some normed linear space. 
have $Q=\{w, x, y, z\}$ and positive numbers $a, b, c$, and $d$ such that $(\alpha) a f_{w}+b f_{x}=c f_{y}+d f_{z}$. Evaluating $(\alpha)$ at $w$ and then at $x$, adding the results and using the triangle inequality, we obtain $(\beta)(a+b) x w$ $=c(x y+y w)+d(x z+z w) \geqq(c+d) x w$. And similarly (evaluating $(\alpha)$ at $y$ and then at $z),(\gamma)(c+d) y z=a(y w+w z)+b(y x+x z) \geqq(a+b) y z$. But $(\beta)$ implies $c+d \leqq a+b$ and $(\gamma)$ implies $a+b \leqq c+d$, so $a+b=c+d$ and the inequalities in $(\beta)$ and $(\gamma)$ can be replaced by equalities, whence we see at once that each triple in $Q$ is linear.

An easy corollary of $(7)$ is

(8) A quadruple $Q$ in a metric space $M$ is metrically dependent in $M$ if and only if $Q$ is pseudo-linear and $p q_{1}+p q_{3}=p q_{2}+p q_{4}$ for each $p \in M$ (where the labelling is as in (ii) of (7)).

\section{REFERENCES}

1. Stefan Banach, Théorie des operations linéaires, Warsaw, 1932.

2. Leonard M. Blumenthal, Theory and applications of distance geometry, Oxford, 1953.

3. C. H. Dowker, An imbedding theorem for paracompact metric spaces, Duke Math. J. vol. 14 (1947) pp. 639-645.

4. C. Kuratowski, Quelques problemes concernant les espaces métriques nonseparables, Fund. Math. vol. 25 (1935) pp. 534-545.

5. I. J. Schoenberg, On certain metric spaces arising from Euclidean spaces by a change of metric and their imbedding in Hilbert space, Ann. of Math. vol. 38 (1937) pp. 787-793.

6. A. H. Stone, Paracompactness and product spaces, Bull. Amer. Math. Soc. vol. 54 (1948) pp. 977-982.

UNiversity of LEIDEN,

UNIVERSITY OF MisSOURI, AND

UNIVERSITY OF WASHINGTON 